\title{
Effect of Processing on Total Iron and Zinc, Ionizable Iron, Extractable Zinc, Phytate and Phytate: Mineral Ratios in Pearl Millet
}

\author{
Aparna Thorat ${ }^{1 *}$, Sonali Rawool ${ }^{1}$, Sheetal Ajbani ${ }^{2}$, Ashwini Salvi ${ }^{1}$, Sneha Chheda ${ }^{2}$, Padmini S. \\ Ghugre $^{3}$, Shobha A. Udipi ${ }^{4}$, Erick Boy ${ }^{5}$ \\ ${ }^{1}$ Research Assistant, Department of Food Science and Nutrition, SNDT Women's University, Mumbai, India \\ ${ }^{2}$ Master Student, Department of Food Science and Nutrition, SNDT Women's University, Mumbai, India \\ ${ }^{3}$ Associate Professor \& Head, Department of Food Science and Nutrition, SNDT Women's University, India \\ ${ }^{4}$ Former Professor \& Head, Department of Food Science and Nutrition, SNDT Women's University, Mumbai, \\ India \\ ${ }^{5}$ Nutrition Co-ordinator, Harvest Plus, IFPRI, Washington \\ Email: aparnathorat88@gmail.com
}

\begin{abstract}
Pearl millet [Pennisetum glaucum (L) R. Br] is a good source of essential minerals but their availability is constrained by high amounts of phytate. Hence, this study examined the effect of soaking, germination and malting on phytate content, phytate: mineral molar ratios, ionizability of iron and extractability of zinc in five varieties of pearl millet namely MAHYCO 204, ICTP 8203, H6M33, Mahalakshmi 504, 8203 Nirmal - Mahabeej. Grain samples were soaked for $12 \mathrm{hrs}$ and $24 \mathrm{hrs}$ in grain: water ratios 1:2 and 1:5 and germinated for $48 \mathrm{hrs}$ after soaking for $12 \mathrm{hrs}$ in grain: water ratios 1:2 and 1:5 and germinated grains were roasted for 6 min for malting. All three processing techniques significantly reduced phytate content compared to the raw. Phytate content was significantly and negatively correlated with ionizable iron $(\mathrm{r}=-0.545)$, percent ionizable iron $(\mathrm{r}=$ $0.653)$, extractable zinc $(\mathrm{r}=-0.214)$, percent extractable zinc $(\mathrm{r}=-0.749)$, phytate: iron $(\mathrm{r}=-$ $0.614)$ and phytate: zinc $(\mathrm{r}=-0.631)$ molar ratios. Mean phytate: iron molar ratio reduced from 15.2 in the raw to 7.6-10.9 on germination and 8.2-8.7 on malting and mean phytate: zinc molar ratio from 19.4 in the raw to $9.4-14.9$ on germination and $11.3-11.8$ on malting.
\end{abstract}

Keywords: Phytate; ionizable iron; extractable zinc; phytate: mineral molar ratios; germination; malting

\section{Introduction}

Pearl millet [Pennisetum glaucum (L.)R. Br] can be cultivated in difficult growing conditions such as drought, low soil fertility and high temperature. Compared to other major cereals it contains higher amounts of calcium, iron, zinc and its protein quality is better [1]. Hence, this millet has the potential for controlling micronutrient deficiencies with specific reference to iron and zinc. However, it contains high amounts of phytate that chelates minerals and reduces their bioavailability.

Several reports in the literature have indicated that phytate content can be reduced by soaking $[2,3]$ and germination $[4,5]$. However, few studies have examined the influence of these processing techniques on the ionizable iron and extractable zinc content as well as the phytate: mineral ratios. The latter is especially relevant to the bioavailability of iron and zinc. Therefore, it is important to determine whether processing can bring the phytate: mineral ratio to the appropriate level as well as improve the ionizability /extractability of iron and zinc.

In Western India, pearl millet is a staple and contributes substantially to the total energy, protein and iron intakes. A survey conducted by this Department on rural adult women in Ahmednagar district, Maharashtra state, India revealed that they consumed about $240 \mathrm{~g}$ of pearl millet per day, which contributed $19.1 \%$ of daily energy intake, $21.5 \%$ of protein intake and about half of the iron and zinc intakes [Dietary Contribution of Pearl Millet in Ahmednagar District, Maharashtra: Consumption and Effect of Traditional Processing on Mineral Bioavailability (2013) Report submitted to Harvest Plus]. In 
this context, we analyzed five pearl millet varieties commonly cultivated and consumed within this region. The effect of selected processing techniques on the in vitro availability of these minerals and the phytate: mineral ratios were examined.

\section{$2 \quad$ Materials and Methods}

\subsection{Sample Collection}

Five varieties of pearl millet (MAHYCO 204, ICTP 8203, H6M33, Mahalakshmi 504, 8203 Nirmal Mahabeej) commonly cultivated in Ahmednagar district in Northwestern region of Maharashtra state, India, were analyzed. Samples were procured directly from farmers. For each variety, 5 to 8 samples were purchased in duplicate from 4-6 villages. In each village, farmers were selected by random sampling from the list of households. All sacks were numbered serially by farmers and from these, two sacks were randomly selected. From each sack three kilograms of pearl millet was purchased by taking one kilogram each from the top, the middle and the bottom portion using an aluminum scoop. The grain was pooled and mixed thoroughly, sealed in plastic Ziplock bags, labeled and transported to the Department in Mumbai city. In the laboratory, the samples were carefully cleaned manually to remove broken grains, stones, chaff and dirt and were stored in fresh clean Ziplock bags.

Sampling of the pearl millet grains was done by quartering method, using stainless steel trays that were washed and rinsed thoroughly with deionized water. Four samples of 300 grams each were taken for each variety. Three samples were kept for studying the effect of processing. The remaining sample was ground to fine flour in a stainless steel mill (mesh size $0.5 \mathrm{~mm}$ ).

\section{$2.2 \quad$ Processing Methods}

Three processing methods were studied - soaking, germination and malting.

Soaking - $30 \mathrm{~g}$ of grains of each sample were accurately weighed in duplicate. The grains were soaked in deionized water, using two grain: water ratios - 1:2 and 1:5 (w/v) and kept at ambient temperature in acid - washed conical flasks for 12 hours and 24 hours, each. After soaking, the excess water was drained and the grains were dried at $60^{\circ} \mathrm{C}$ to constant weight. The dried seeds were ground to flour (mesh size $0.5 \mathrm{~mm}$ ) in a stainless steel mill.

Germination - 30g of grains were soaked in deionized water using the grain: water ratios mentioned above and kept at ambient temperature $\left(35-40^{\circ} \mathrm{C}\right)$ for 12 hours. After soaking, the water was drained and soaked grains were covered with a muslin cloth, allowed to germinate at ambient temperature for 48 hours, were dried to constant weight and ground to obtain the germinated millet flour $[2,3,4,5]$.

Malting- $30 \mathrm{~g}$ of grains were germinated as described above. The germinated dried grains were roasted for 6 minutes in a thick-bottomed stainless steel pan that had been rinsed with deionized water. After malting, the grains were ground to obtain the malted millet flour.

The flours obtained using the three processing techniques as well as the raw grain flour were packed in plastic bags, sealed and packed in air tight plastic boxes and stored at $4^{\circ} \mathrm{C}$ until analysis.

\subsection{Chemicals}

All chemicals were used according to analytical grade. Pepsinand thioglycollic acid was purchased from Loba chemie Laboratory reagents and fine chemicals. Hydrochloric acid, perchloric acid, nitric acid, acetic acid glacial, 2,2 -Bipyridyl, sodium acetate anhydrous, hydroxylamine hydrochloride, ammonium ferric sulfate, ammonium ferrous sulfate, iron and zinc standard solutions were procured from Merk Specialities Pvt. Ltd, Mumbai, India.Phytic acidsodium salt hydrate standard (Pcode-100969894) was procured from Sigma-Aldrich Co., St. Louis, USA.

\subsection{Analytical Methods}

For each flour raw and processed, samples were drawn in duplicate and each sample was then analyzed in triplicate. Total zinc and iron: Two grams of each sample was subjected to dry ashing at $550^{\circ} \mathrm{C}$ in a 
muffle furnace as per AOAC method [6] and ash solutions were prepared using deionized water. In these ash solutions, total iron and zinc content were determined by Inductively Coupled Plasma Optical Emission Spectrometry (Perkin Elmer Model Optima 7000 DV, Shelton, CT, USA) in ash solutions.

Phytate: Phytate content was estimated Spectrophotometrically (LABINDIA Model UV3000+, Navi Mumbai, India) using the procedure given by Haug and Lantzsch [7] at 519nm.

Ionizable iron: Ionizable iron content was estimated at $\mathrm{pH} 7.5$ by the method of Rao and Prabhavathi [8].

Extractable zinc: Extractable zinc content was estimated by the method given by Chompreeda and Fields [9].

\subsection{Data Analysis}

Molar ratios of phytate:iron and phytate:zinc were calculated. SPSS (version 17.0) was used for statistical analysis. One - way Analysis of Variance (ANOVA) followed by Bonferroni post - hoc test for multiple comparisons was used to determine whether differences among the raw and processed samples were significant. Pearson's correlation analysis was used to examine the relationship between phytate, ionizable iron and extractable zinc.

\section{$3 \quad$ Results}

\subsection{Raw Samples}

Iron and Zinc Content: Overall mean content of iron and zinc was $5.1 \mathrm{mg} \%$ and $4.9 \mathrm{mg} \%$, respectively (Table 1). Significant differences were seen between the five varieties for total iron $(F=$ 3.072, $\mathrm{p}=0.021)$ and total zinc $(\mathrm{F}=7.402, \mathrm{p}=0.000)$, with ICTP-8203 having the highest content of iron and zinc, among the five varieties. Mahalakshmi 504 and H6M33 had the lowest iron content while zinc content was least in Mahalakshmi 504.

Phytate Content: The overall mean phytate content was $878 \mathrm{mg} \%$ (Table 1), with significant differences between the five varieties $(\mathrm{F}=4.024, \mathrm{p}=0.009)$, H6M33 having the highest phytate content and 8203-Mahabeej/Nirmal the lowest content, followed by ICTP-8203.

Phytate: mineral ratios: The phytate:iron molar ratio varied from 8.6 to 20.9, with significant varietal differences $(\mathrm{F}=40.058, \mathrm{p}=0.000)$. Similar significant varietal differences were seen in the phytate:zinc molar ratio which ranged from 9.6 to 36.9 (Table 1).

Ionizable Iron and Extractable Zinc Content: Mean ionizable iron for all five varieties was $0.7 \pm 0.2 \mathrm{mg} \%$ and mean extractable zinc was $2.5 \pm 0.7 \mathrm{mg} \%$, with significant varietal differences for both ionizable iron $(\mathrm{F}=31.329, \mathrm{p}=0.000)$ and extractable zinc $(\mathrm{F}=4.177, \mathrm{p}=0.007)$. ICTP-8203 had the highest whereas H6M33 had the lowest ionizable iron content. Mahalakshmi-504 had the lowest amount of extractable zinc in contrast to ICTP-8203 which had the highest content of extractable zinc. Ionizable iron as a percentage of total iron on average, constituted only $14.1 \pm 4.3 \%$. However, extractable zinc constituted almost half of the total zinc $(51.2 \pm 7.8 \%)$.

Table 1. Total and ionizable iron, total and extractable zinc, phytate content and phytate: mineral molar ratios in raw pearl millet $(\mathrm{mg} / 100 \mathrm{gm} \mathrm{DM})$

\begin{tabular}{|c|c|c|c|c|c|c|}
\hline \multirow[t]{2}{*}{ Raw } & \multicolumn{5}{|c|}{ Variety } & \multirow[t]{2}{*}{ Overal } \\
\hline & 204 & ICTP 8203 & H6M33 & $\begin{array}{c}\text { 8203-Mahabeej/ } \\
\text { Nirmal }\end{array}$ & Mahalakshmi & \\
\hline \multicolumn{7}{|c|}{ Total Iron } \\
\hline Mean \pm SD & $5.4 \pm 1.3$ & 5. $7 \pm 0.9$ & $4.4 \pm 0.1$ & $4.7 \pm 0.6$ & $4.4 \pm 0.2$ & $5.1 \pm 1.0$ \\
\hline Min-Max & $3.1-8.1$ & $4.9-7.7$ & $4.2-4.5$ & $3.9-5.6$ & $4.1-4.6$ & $3.9-8.1$ \\
\hline \multicolumn{7}{|c|}{ Total Zinc } \\
\hline Mean \pm SD & $5.2 \pm 1.0$ & $6.0 \pm 0.9$ & $4.0 \pm 0.8$ & $4.8 \pm 1.4$ & $3.2 \pm 0.9$ & $4.9 \pm 1.3$ \\
\hline Min-Max & $4.0-6.8$ & $4.1-6.9$ & $3.4-5.5$ & $3.7-7.0$ & $2.4-4.5$ & $2.4-7.0$ \\
\hline
\end{tabular}




\begin{tabular}{c|c|c|c|c|c|c}
\hline \multicolumn{7}{c}{ Phytate } \\
\hline Mean \pm SD & $9256 \pm 105$ & $833 \pm 137$ & $952 \pm 84$ & $763 \pm 53$ & $868 \pm 64$ & $878 \pm 115$ \\
\hline Min-Max & $772-1091$ & $667-1036$ & $828-1041$ & $676-815$ & $789-950$ & $667-1091$ \\
\hline \multicolumn{7}{c}{ Phytate:Iron Molar ratio } \\
\hline Mean \pm SD & $15.2 \pm 3.2$ & $12.6 \pm 2.4$ & $18.4 \pm 1.6$ & $13.9 \pm 1.8$ & $16.9 \pm 1.3$ & $15.2 \pm 3.0$ \\
\hline Min-Max & $9.2-20.9$ & $8.6-16.1$ & $15.9-19.9$ & $11.7-17.3$ & $15.5-18.5$ & $8.6-20.9$ \\
\hline \multicolumn{7}{c}{ Phytate:Zinc Molar ratio } \\
\hline Mean \pm SD & $18.2 \pm 3.5$ & $14.3 \pm 4.7$ & $24.2 \pm 5.3$ & $16.6 \pm 4.4$ & $28.6 \pm 8.6$ & $19.4 \pm 6.7$ \\
\hline Min-Max & $13.1-24.1$ & $9.6-24.7$ & $14.7-29.8$ & $10.8-21.2$ & $17.4-36.9$ & $9.6-36.9$ \\
\hline \multicolumn{7}{|c|}{ Ionizable iron } \\
\hline Mean \pm SD & $0.7 \pm 0.1$ & $1.0 \pm 0.1$ & $0.4 \pm 0.1$ & $0.8 \pm 0.1$ & $0.6 \pm 0.1$ & $0.7 \pm 0.2$ \\
\hline Min-Max & $0.5-0.8$ & $0.9-1.1$ & $0.3-0.4$ & $0.6-0.9$ & $0.5-0.8$ & $0.3-1.1$ \\
\hline \multicolumn{7}{c}{ Extractable Zinc } \\
\hline Mean \pm SD & $2.4 \pm 0.4$ & $3.1 \pm 0.9$ & $2.2 \pm 0.5$ & $2.7 \pm 0.8$ & $1.8 \pm 0.5$ & $2.5 \pm 0.7$ \\
\hline Min-Max & $1.9-3.4$ & $1.7-4.1$ & $1.9-3.2$ & $2.0-4.1$ & $1.4-2.5$ & $1.4-4.1$ \\
\hline
\end{tabular}

\subsection{Effect of Processing}

Soaking: Mean content of total iron and zinc decreased upon soaking with all four treatments i.e. 12 and 24 hours of soaking and grain: water ratios of 1:2 and 1:5 (Table 2). This decrease was statistically significant for iron $(\mathrm{F}=22.435, \mathrm{p}=0.000)$ but not for zinc $(\mathrm{F}=0.990, \mathrm{p}=0.440)$.

Iron content decreased as the duration of soaking increased for all varieties at both the grain: water ratios. In grains soaked for $12 \mathrm{hrs,} \mathrm{iron} \mathrm{content} \mathrm{was} \mathrm{slightly} \mathrm{but} \mathrm{not} \mathrm{significantly} \mathrm{higher} \mathrm{with} \mathrm{grain:}$ water ratio of 1:5 than with a ratio of 1:2. With 24 hours of soaking, there was little difference between the two ratios. Percent reduction in total iron content after 12 hours of soaking was $28.2 \%$, with grain: water ratio of 1:2 and $18.9 \%$ with the higher grain: water ratio (1:5). With 24 hours of soaking, about one-third $(36.5 \%)$ of the iron was lost with grain: water ratio of $1: 2$ and $39.7 \%$ with grain: water ratio of 1:5. In case of zinc, duration of soaking and grain: water ratios did not significantly influence the zinc content. Soaking for 12 hours resulted in $29.8 \%$ loss with grain: water ratio of $1: 2$ and $36.4 \%$ with grain: water ratio of $1: 5$.

The extent of loss in total iron content differed significantly between varieties for all four treatments. Overall, ICTP 8203 showed greater losses than the other varieties, especially after 24 hours of soaking. Similar trends were observed for loss of total zinc.

Phytate content of soaked samples was significantly lower than that of raw samples for all five varieties (Table 2). There was a significant difference between the four treatments i.e. duration of soaking and the two grain: water ratios $(\mathrm{F}=75.057, \mathrm{p}=0.000)$. Comparison of the five varieties using post-hoc Bonferroni test indicated significant differences for each of the four treatments $(\mathrm{p}<0.001)$ with the extent of loss on soaking being $23 \%$ for MAHYCO 204, $26.9 \%$ for ICTP-8203, 31.4\% for H6M33, $32.5 \%$ in 8203 - Mahabeej-Nirmal and highest, i.e. $39.9 \%$ for Mahalakshmi.

The phytate: iron molar ratio in the soaked samples was similar to the ratio present in the raw grains (Table 3). Significant varietal differences were observed for the phytate: iron ratio $(\mathrm{F}=40.058, \mathrm{p}=0.000)$, the lowest being in ICTP 8203 and 8203 - Mahabeej-Nirmal and the highest in MAHYCO 204. Similarly, significant varietal differences were seen in the phytate: zinc molar ratio $(\mathrm{F}=23.539, \mathrm{p}=0.000)$, the lowest in 8203 - Mahabeej-Nirmal and the highest in H6M33.

There was no significant difference in the overall ionizable iron content of soaked and raw samples (Table 3). Ionizable iron expressed as a percentage of total iron, was higher in the soaked grains than raw grains, for all varieties, ranging from 20.3 to $25.7 \%$ (Figure 1). However, varietal differences were observed. In three varieties, namely, MAHYCO 204, ICTP-8203 and 8203 - Mahabeej-Nirmal, ionizable iron content in the soaked samples was similar to that of the raw samples, regardless of amount of water used and duration of soaking. In contrast, in H6M33 and Mahalakshmi 504, the ionizable iron content was higher in soaked samples than in raw samples (Table 3).

The extractable zinc content decreased slightly after soaking (Table 3). After 12 hours of soaking, with a grain: water ratio of $1: 2$, mean extractable zinc was slightly but not significantly lower than in 
raw samples. When more water was used for soaking (grain:water ratio of 1:5), the mean extractable zinc was similar to the content found with grain: water ratio of 1:2. When the duration of soaking was extended to 24 hours, there was no further decrease at both the ratios of grain to water (Table 3). When the extractable zinc as a percentage of total zinc was examined, the values $(58.8 \%$ to $66.6 \%)$ were higher for soaked samples, with all four treatments, than in the raw samples (Figure 2). Comparison of the four soaking treatments showed that with a grain: water ratio of 1:5 and 24 hours duration of soaking, the extractable zinc constituted about $60 \%$ of the total zinc (Figure 2). Varietal differences were not observed for extractable zinc.

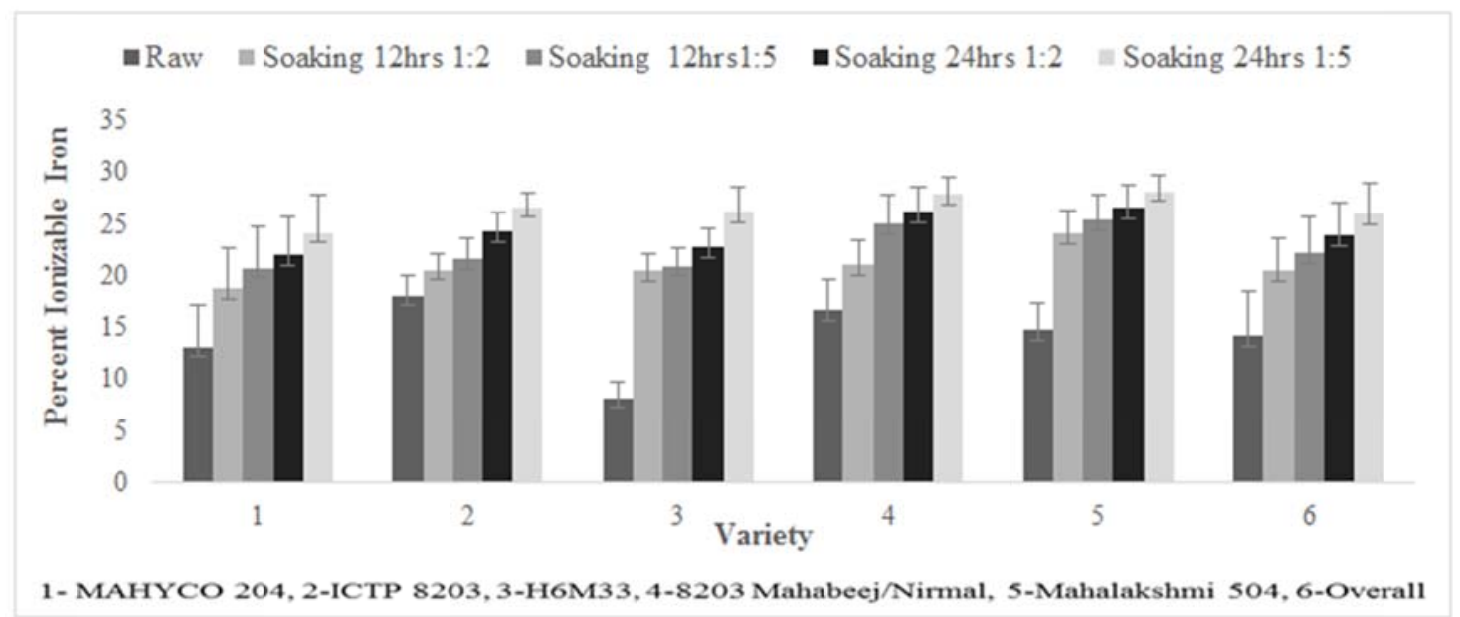

Figure 1. Effect of soaking on percent ionizable iron in five varieties of pearl millet

Table 2. Total iron, total zinc and phytate content in soaked pearl millet (mg/100gm DM)

\begin{tabular}{|c|c|c|c|c|c|c|c|}
\hline \multirow[t]{2}{*}{ Parameter } & \multirow{2}{*}{$\begin{array}{c}\text { Hours, } \\
\text { Grain: Water } \\
\text { Ratio } \\
\end{array}$} & \multicolumn{5}{|c|}{ Variety } & \multirow[t]{2}{*}{ Overall } \\
\hline & & $\begin{array}{c}\text { MAHYCO } \\
204 \\
\end{array}$ & $\begin{array}{r}\text { ICTP } \\
8203 \\
\end{array}$ & H6M33 & $\begin{array}{c}\text { 8203-Mahabeej / } \\
\text { Nirmal }\end{array}$ & Mahalakshmi & \\
\hline \multicolumn{8}{|c|}{ Total Iron - 12 hrs } \\
\hline Mean \pm SD & \multirow[t]{2}{*}{$1: 2$} & $3.6 \pm 0.5$ & $4.2 \pm 0.3$ & $3.2 \pm 0.3$ & $3.7 \pm 0.3$ & $3.2 \pm 0.2$ & $3.6 \pm 0.5$ \\
\hline Min-Max & & $3.1-4.8$ & $3.8-4.9$ & $2.8-3.7$ & $3.5-4.2$ & $3.1-3.4$ & $2.8-4.9$ \\
\hline Mean \pm SD & \multirow[t]{2}{*}{$1: 5$} & $4.0 \pm 0.6$ & $4.6 \pm 0.3$ & $3.8 \pm 0.3$ & $4.1 \pm 0.7$ & $3.8 \pm 0.2$ & $4.0 \pm 0.5$ \\
\hline Min-Max & & $3.3-5.6$ & $4.3-5.3$ & $3.4-4.2$ & $3.1-5.0$ & $3.6-4.1$ & $3.1-5.6$ \\
\hline \multicolumn{8}{|c|}{ Total Iron - $24 \mathrm{hrs}$} \\
\hline Mean \pm SD & \multirow[t]{2}{*}{$1: 2$} & $3.5 \pm 0.5$ & $3.3 \pm 0.3$ & $2.9 \pm 0.4$ & $2.8 \pm 0.4$ & $3.0 \pm 0.3$ & $3.2 \pm 0.5$ \\
\hline Min-Max & & $2.6-4.3$ & $2.8-3.7$ & $2.3-3.6$ & $2.4-3.6$ & $2.7-3.3$ & $2.3-4.3$ \\
\hline Mean \pm SD & \multirow[t]{2}{*}{$1: 5$} & $3.5 \pm 0.6$ & $3.0 \pm 0.3$ & $2.5 \pm 0.3$ & $2.7 \pm 0.2$ & $2.9 \pm 0.3$ & $3.0 \pm 0.6$ \\
\hline Min-Max & & $2.6-4.4$ & $2.41-3.20$ & $2.1-2.9$ & $2.5-3.2$ & $2.6-3.4$ & $2.0-4.4$ \\
\hline \multicolumn{8}{|c|}{ Total Zinc - 12 hrs } \\
\hline Mean \pm SD & \multirow[t]{2}{*}{$1: 2$} & $3.8 \pm 0.7$ & $3.4 \pm 0.5$ & $2.4 \pm 0.3$ & $4.0 \pm 0.6$ & $2.9 \pm 0.3$ & $3.4 \pm 0.8$ \\
\hline Min-Max & & $2.9-5.0$ & $2.7-4.10$ & $2.1-2.8$ & $3.1-4.9$ & $2.5-3.2$ & $2.1-5.0$ \\
\hline Mean \pm SD & \multirow[t]{2}{*}{$1: 5$} & $3.8 \pm 0.7$ & $3.4 \pm 0.3$ & $3.0 \pm 0.3$ & $3.3 \pm 0.3$ & $3.0 \pm 0.5$ & $3.4 \pm 0.6$ \\
\hline Min-Max & & $3.0-4.9$ & $2.6-3.7$ & $2.5-3.4$ & $2.9-3.6$ & $2.3-3.5$ & $2.3-4.9$ \\
\hline \multicolumn{8}{|c|}{ Total Zinc - $24 \mathrm{hrs}$} \\
\hline Mean \pm SD & \multirow[t]{2}{*}{$1: 2$} & $3.8 \pm 0.8$ & $2.9 \pm 0.7$ & $3.1 \pm 0.9$ & $2.8 \pm 0.4$ & $2.4 \pm 0.3$ & $3.2 \pm 0.9$ \\
\hline Min-Max & & $3.0-5.8$ & $2.1-4.2$ & $2.2-4.5$ & $2.5-3.6$ & $2.1-2.8$ & $2.1-5.8$ \\
\hline Mean \pm SD & \multirow[t]{2}{*}{$1: 5$} & $3.3 \pm 0.8$ & $2.9 \pm 0.4$ & $2.5 \pm 0.3$ & $3.2 \pm 0.5$ & $2.8 \pm 0.3$ & $3.0 \pm 0.6$ \\
\hline Min-Max & & $2.1-4.9$ & $2.5-3.5$ & $2.3-3.0$ & $2.7-3.9$ & $2.3-3.1$ & 2.1-4.9 \\
\hline & & & Phyt: & $-12 \mathrm{hrs}$ & & & \\
\hline
\end{tabular}




\begin{tabular}{|c|c|c|c|c|c|c|c|}
\hline Mean \pm SD & \multirow[t]{2}{*}{$1: 2$} & $718 \pm 87$ & $657 \pm 105$ & $702 \pm 80$ & $573 \pm 39$ & $548 \pm 79$ & $659 \pm 103$ \\
\hline Min-Max & & $611-898$ & $545-836$ & $549-770$ & $535-636$ & $444 \pm 631$ & $444-898$ \\
\hline Mean \pm SD & \multirow[t]{2}{*}{$1: 5$} & $676 \pm 94$ & $610 \pm 84$ & $697 \pm 74$ & $539 \pm 43$ & $545 \pm 74$ & $628 \pm 98$ \\
\hline Min-Max & & $563 \pm 857$ & $492-768$ & $625-809$ & $468-588$ & $435-616$ & $435-857$ \\
\hline \multicolumn{8}{|c|}{ Phytate - 24 hrs } \\
\hline Mean \pm SD & \multirow[t]{2}{*}{$1: 2$} & $702 \pm 67$ & $607 \pm 159$ & $643 \pm 52$ & $489 \pm 85$ & $599 \pm 54$ & $627 \pm 114$ \\
\hline Min-Max & & $537-793$ & $344-804$ & $574-695$ & $333-586$ & $542-674$ & $333-804$ \\
\hline Mean \pm SD & \multirow[t]{2}{*}{$1: 5$} & $656 \pm 75$ & $562 \pm 59$ & $571 \pm 98$ & $458 \pm 41$ & $393 \pm 66$ & $560 \pm 115$ \\
\hline Min-Max & & $545-786$ & $4667-630$ & $422-714$ & $398-517$ & $322-464$ & $322-786$ \\
\hline
\end{tabular}

Table 3. Phytate: mineral ratios, ionizable iron and extractable zinc content in soaked pearl millet (mg/100gm DM)

\begin{tabular}{|c|c|c|c|c|c|c|c|}
\hline \multirow[t]{2}{*}{ Parameter } & \multirow{2}{*}{\begin{tabular}{|c|} 
Hours, \\
Grain: Water \\
Ratio
\end{tabular}} & \multicolumn{5}{|c|}{ Variety } & \multirow[t]{2}{*}{ Overall } \\
\hline & & $\begin{array}{c}\text { MAHYCO } \\
204\end{array}$ & $\begin{array}{c}\text { ICTP } \\
8203\end{array}$ & H6M33 & $\begin{array}{c}\text { 8203-Mahabeej } / \\
\text { Nirmal }\end{array}$ & Mahalakshmi & \\
\hline \multicolumn{8}{|c|}{ Phytate : Iron Molar Ratio - 12 hrs } \\
\hline Mean \pm SD & \multirow[t]{2}{*}{$1: 2$} & $17.0 \pm 3.2$ & $13.5 \pm 2.9$ & $18.8 \pm 2.5$ & $13.1 \pm 0.7$ & $14.5 \pm 2.5$ & $15.7 \pm 3.3$ \\
\hline Min-Max & & $13.1-23.3$ & $8.6-16.1$ & $15.9-23.3$ & $12.4-13.9$ & $12.1-17.4$ & $11.1-23.3$ \\
\hline Mean \pm SD & \multirow[t]{2}{*}{$1: 5$} & $14.6 \pm 2.9$ & $11.2 \pm 1.7$ & $15.7 \pm 2.4$ & $11.3 \pm 1.6$ & $12.2 \pm 2.2$ & $13.3 \pm 2.9$ \\
\hline Min-Max & & $8.6-19.9$ & $8.8-14.8$ & $12.7-19.9$ & $9.3-13.0$ & $9.4-14.4$ & $8.6-19.9$ \\
\hline \multicolumn{8}{|c|}{ Phytate : Iron Molar Ratio - 24 hrs } \\
\hline Mean \pm SD & \multirow[t]{2}{*}{$1: 2$} & $17.3 \pm 3.1$ & $16.1 \pm 4.7$ & $19.0 \pm 3.1$ & $15.2 \pm 3.9$ & $16.9 \pm 2.7$ & $16.9 \pm 3.6$ \\
\hline Min-Max & & $12.1-22.3$ & $8.5-21.9$ & $14.6-22.5$ & $7.9-19.1$ & $13.8-21.3$ & $7.9-22.5$ \\
\hline Mean \pm SD & \multirow[t]{2}{*}{$1: 5$} & $16.3 \pm 3.6$ & $16.3 \pm 2.6$ & $19.2 \pm 2.7$ & $14.4 \pm 1.4$ & $11.6 \pm 2.6$ & $15.9 \pm 3.5$ \\
\hline Min-Max & & $11.9-23.9$ & $12.8-20.3$ & $15.6-23.9$ & $12.8-16.0$ & $8.4-14.5$ & $8.4-23.9$ \\
\hline \multicolumn{8}{|c|}{ Phytate : Zinc Molar Ratio - 12 hrs } \\
\hline Mean \pm SD & \multirow[t]{2}{*}{$1: 2$} & $19.0 \pm 3.9$ & $19.5 \pm 4.7$ & $28.8 \pm 3.5$ & $14.6 \pm 2.7$ & $19.1 \pm 4.1$ & $19.9 \pm 5.6$ \\
\hline Min-Max & & $13.9-25.6$ & $13.6-26.4$ & $25.1-33.3$ & $11.3-19.3$ & $14.3-23.5$ & $11.3-33.3$ \\
\hline Mean \pm SD & \multirow[t]{2}{*}{$1: 5$} & $18.2 \pm 4.1$ & $17.9 \pm 3.3$ & $23.6 \pm 4.1$ & $15.9 \pm 0.9$ & $18.5 \pm 3.6$ & $18.6 \pm 4.1$ \\
\hline Min-Max & & $13.7-28.2$ & $13.2-23.8$ & $18.1-30.6$ & $14.5-16.9$ & $14.3-24.2$ & $13.2-30.6$ \\
\hline \multicolumn{8}{|c|}{ Phytate : Zinc Molar Ratio - 24 hrs } \\
\hline Mean \pm SD & \multirow[t]{2}{*}{$1: 2$} & $18.6 \pm 3.5$ & $22.2 \pm 8.8$ & $21.8 \pm 5.2$ & $17.5 \pm 4.5$ & $24.6 \pm 1.9$ & $20.4 \pm 5.5$ \\
\hline Min-Max & & $11.6-25.1$ & $11.8-34.8$ & $14.7-27.7$ & $9.1-21.6$ & $22.1-27.4$ & $9.1-34.2$ \\
\hline Mean \pm SD & \multirow[t]{2}{*}{$1: 5$} & $20.8 \pm 5.3$ & $19.1 \pm 2.1$ & $22.4 \pm 3.0$ & $14.1 \pm 1.6$ & $13.9 \pm 2.9$ & $18.8 \pm 4.8$ \\
\hline Min-Max & & $14.9-33.9$ & $15.3-21.8$ & $17.5-26.3$ & $11.5-15.9$ & $10.9-17.2$ & $10.9-33.9$ \\
\hline \multicolumn{8}{|c|}{ Ionizable Iron - $12 \mathrm{hrs}$} \\
\hline Mean \pm SD & \multirow[t]{2}{*}{$1: 2$} & $0.7 \pm 0.1$ & $0.9 \pm 0.0$ & $0.7 \pm 0.1$ & $0.8 \pm 0.1$ & $0.8 \pm 0.1$ & $0.7 \pm 0.1$ \\
\hline Min-Max & & $0.5-0.9$ & $0.7-1.0$ & $0.5-0.8$ & $0.7-0.9$ & $0.7-0.8$ & $0.5-1.0$ \\
\hline Mean \pm SD & \multirow[t]{2}{*}{$1: 5$} & $0.8 \pm 0.2$ & $1.00 \pm 0.1$ & $0.8 \pm 0.1$ & $1.0 \pm 0.1$ & $1.0 \pm 1.0$ & $0.9 \pm 0.2$ \\
\hline Min-Max & & $0.6-1.1$ & $0.9-1.1$ & $0.6-1.0$ & $0.8-1.1$ & $0.9-1.0$ & $0.6-1.1$ \\
\hline \multicolumn{8}{|c|}{ Ionizable Iron - $24 \mathrm{hrs}$} \\
\hline Mean \pm SD & \multirow[t]{2}{*}{$1: 2$} & $0.8 \pm 0.1$ & $0.8 \pm 0.1$ & $0.7 \pm 0.1$ & $0.7 \pm 0.1$ & $0.8 \pm 0.1$ & $0.8 \pm 0.1$ \\
\hline Min-Max & & $0.5-1.0$ & $0.6-0.9$ & $0.5-0.8$ & $0.6-0.9$ & $0.7-1.0$ & $0.5-1.0$ \\
\hline Mean \pm SD & \multirow[t]{2}{*}{$1: 5$} & $0.8 \pm 0.1$ & $0.8 \pm 0.1$ & $0.7 \pm 0.1$ & $0.8 \pm 0.1$ & $0.8 \pm 0.1$ & $0.8 \pm 0.1$ \\
\hline Min-Max & & $0.6-1.0$ & $0.6-0.9$ & $0.6-0.7$ & $0.6-0.9$ & $0.7-0.9$ & $0.6-1.0$ \\
\hline \multicolumn{8}{|c|}{ Extractable zinc - $12 \mathrm{hrs}$} \\
\hline Mean \pm SD & $1: 2$ & $2.2 \pm 0.3$ & $1.9 \pm 0.3$ & $1.5 \pm 0.2$ & $2.4 \pm 0.4$ & $1.8 \pm 0.2$ & $2.0 \pm 0.4$ \\
\hline Min-Max & & $1.7-2.7$ & $1.4-2.5$ & $1.3-1.8$ & $1.8-3.0$ & $1.5-1.9$ & $1.3-3.0$ \\
\hline Mean \pm SD & $1: 5$ & $2.3 \pm 0.4$ & $2.1 \pm 0.3$ & $1.9 \pm 0.3$ & $2.2 \pm 0.2$ & $1.9 \pm 0.3$ & $2.1 \pm 0.4$ \\
\hline Min-Max & & $1.8-3.1$ & $1.6-2.4$ & $1.6-2.2$ & $1.9-2.4$ & $1.5-2.3$ & $1.5-3.1$ \\
\hline
\end{tabular}




\begin{tabular}{c|c|c|c|c|c|c|c}
\hline \multicolumn{9}{c}{ Extractable zinc - 24 hrs } \\
\hline Mean \pm SD & $\mathbf{1 : 2}$ & $2.4 \pm 0.4$ & $1.9 \pm 0.5$ & $2.1 \pm 0.6$ & $1.9 \pm 0.2$ & $1.7 \pm 0.1$ & $2.1 \pm 0.5$ \\
\hline Min-Max & & $1.9-3.6$ & $1.3-2.8$ & $1.5-3.1$ & $1.6-2.2$ & $1.6-1.8$ & $1.3-3.6$ \\
\hline Mean \pm SD & $\mathbf{1 : 5}$ & $2.1 \pm 0.5$ & $2.0 \pm 0.3$ & $1.7 \pm 0.2$ & $2.1 \pm 0.2$ & $1.9 \pm 0.1$ & $2.0 \pm 0.4$ \\
\hline Min-Max & & $1.3-2.9$ & $1.5-2.4$ & $1.5-2.1$ & $1.9-2.5$ & $1.8-2.1$ & $1.3-2.9$ \\
\hline
\end{tabular}

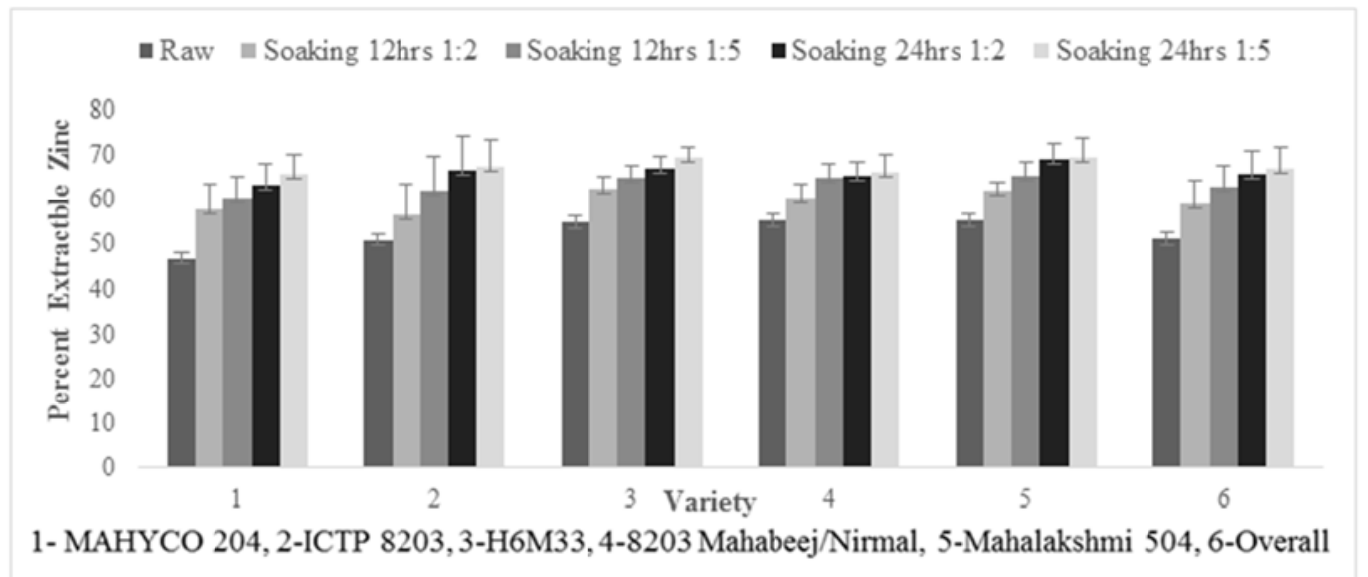

Figure 2. Effect of soaking on percent extractable zinc in five varieties of pearl millet

Germination: Germinated grains had a significantly lower iron and zinc content $(\mathrm{p}<0.001)$ than the raw grains (Table 4). Percent decrease in total iron was $17.5 \%$ when the grain: water ratio was $1: 2$, whereas when the ratio was 1:5, the decrease was approximately half (8.8\%). Similarly there was a decrease in total zinc content with loss being $31.2 \%$ in samples soaked with a grain: water ratio of 1:2 and $41.7 \%$ when grain: water ratio was 1:5. The total zinc and iron content of germinated grains was similar to that in soaked grains.

The mean phytate content of germinated grains was significantly lower $(\mathrm{p}<0.001)$ than the raw grains for all the varieties, with the decrease being slightly more than $50 \%$ (Table 4 ). The percent decrease was slightly higher when the grain: water ratio was 1:2 than when it was 1:5. Also, the germinated grains had significantly lower phytate content $(\mathrm{p}=0.000)$ than their soaked counterparts.

The phytate: iron molar ratio in germinated grains was significantly lower than in both the raw and soaked samples (Table 4). However, the ratios were slightly higher when the grain: water ratio used for soaking was 1:5 than when it was 1:2. In case of zinc also, the phytate: zinc molar ratio in germinated samples was significantly lower than in the raw and soaked samples. With the 1:5 grain: water ratio, the phytate: zinc molar ratio was higher than that with the 1:2 grain: water ratio.

Ionizable iron content of germinated samples was significantly higher compared to the raw samples, but did not differ significantly from the soaked samples. As a percentage of total iron, the ionizable iron content was approximately $27 \%$ (Figure 3). Extractable zinc constituted about $70 \%$ of total zinc in the germinated samples (Figure 4). Compared to the raw and soaked grains, extractable zinc content in the germinated samples was only marginally higher.

Malting: In contrast to germination, malting did not significantly affect the mean total iron content for both the grain: water ratios (Table 5). Total zinc content of malted samples was significantly lower than in the raw, however, the content was similar to that in the germinated grains. Malting significantly reduced the phytate content when compared to the raw samples, with the percent decrease being about $61 \%$. However, compared to the germinated samples with 1:2 grain: water ratio, the malted samples had $12.9 \%$ higher phytate content. In contrast, with 1:5 grain: water ratio, there was $19.6 \%$ decrease in phytate content. The phytate: iron molar ratio was about half that observed for the raw and soaked samples, with the difference being statistically significant $(\mathrm{F}=40.058, \mathrm{p}=0.000)$. However, the malted samples did not differ significantly from germinated samples. A similar trend was observed for the phytate: zinc molar ratio $(\mathrm{F}=23.539, \mathrm{p}=0.000)$. 
As against total iron, the ionizable iron content was significantly higher in the malted than in the raw and germinated grains at both the grain: water ratios. Ionizable iron as a percentage of total iron was $43.3 \%$ for the 1:2 grain: water ratio and $52 \%$ for the 1:5 ratio (Figure 3). Extractable zinc as a percentage of total zinc was approximately $80 \%$, which is slightly higher than in the raw and soaked grains (Figure 4).

Correlation between percent ionizable iron, extractable zinc and phytate: Phytate content was negatively and significantly correlated with ionizable iron content $(\mathrm{r}=-0.545, \mathrm{p}=0.000)$, percent ionizable iron $(\mathrm{r}=-0.653, \mathrm{p}=0.000)$, extractable zinc $(\mathrm{r}=-0.214, \mathrm{p}=0.000)$ and percent extractable zinc $(\mathrm{r}=-0.749, \mathrm{p}=0.000)$. Similarly, a significant negative correlation was observed between phytate: iron molar ratio and ionizable iron content $(\mathrm{r}=-0.614, \mathrm{p}=0.000)$ and between phytate: zinc molar ratio and extractable zinc content $(\mathrm{r}=-0.631, \mathrm{p}=0.000)$.

Table 4. Total and ionizable iron, total and extractable zinc and phytate content, phytate: mineral molar ratios in germinated pearl millet (mg/100gm DM)

\begin{tabular}{|c|c|c|c|c|c|c|c|}
\hline \multirow[t]{2}{*}{ Parameter } & \multirow{2}{*}{$\begin{array}{c}\text { Hours, } \\
\text { Grain: Water } \\
\text { Ratio }\end{array}$} & \multicolumn{5}{|c|}{ Variety } & \multirow[t]{2}{*}{ Overall } \\
\hline & & \begin{tabular}{|c|} 
MAHYCO \\
204 \\
\end{tabular} & $\begin{array}{l}\text { ICTP } \\
8203 \\
\end{array}$ & H6M33 & $\begin{array}{c}\text { 8203-Mahabeej / } \\
\text { Nirmal }\end{array}$ & Mahalakshmi & \\
\hline \multicolumn{8}{|c|}{ Total Iron } \\
\hline Mean \pm SD & \multirow[t]{2}{*}{$1: 2$} & $3.7 \pm 1.0$ & $3.7 \pm 0.3$ & $2.8 \pm 0.2$ & $3.3 \pm 0.2$ & $3.1 \pm 0.3$ & $3.4 \pm 0.7$ \\
\hline Min-Max & & $2.5-5.4$ & $3.2-4.1$ & $2.5-2.9$ & $3.0-3.7$ & $2.7-3.4$ & $2.5-5.4$ \\
\hline Mean \pm SD & \multirow[t]{2}{*}{$1: 5$} & $4.2 \pm 1.0$ & $3.6 \pm 0.3$ & $3.0 \pm 0.2$ & $3.1 \pm 0.3$ & $2.9 \pm 0.1$ & $3.5 \pm 0.8$ \\
\hline Min-Max & & $2.8-5.8$ & $3.2-3.9$ & $2.7-3.3$ & $2.6-3.4$ & $2.7-3.0$ & $2.6-5.8$ \\
\hline \multicolumn{8}{|c|}{ Total Zinc } \\
\hline Mean \pm SD & \multirow[t]{2}{*}{$1: 2$} & $3.8 \pm 1.1$ & $3.1 \pm 0.3$ & $2.9 \pm 0.4$ & $3.3 \pm 0.4$ & $2.6 \pm 0.3$ & $3.3 \pm 0.8$ \\
\hline Min-Max & & $2.4-5.4$ & $2.5-3.4$ & $2.5-3.4$ & $2.8-4.0$ & $2.3-3$ & $2.3-5.4$ \\
\hline Mean \pm SD & \multirow[t]{2}{*}{$1: 5$} & $3.7 \pm 1.0$ & $2.9 \pm 0.5$ & $2.5 \pm 0.4$ & $3.6 \pm 0.6$ & $2.4 \pm 0.3$ & $3.1 \pm 0.8$ \\
\hline Min-Max & & $2.3-5.0$ & $2.5-3.8$ & 1.9-3.6 & $2.8-4.2$ & $2.1-2.9$ & $1.9-5.0$ \\
\hline \multicolumn{8}{|c|}{ Phytate } \\
\hline Mean \pm SD & \multirow[t]{2}{*}{$1: 2$} & $239 \pm 102$ & $384 \pm 74$ & $381 \pm 162$ & $251 \pm 8$ & $245 \pm 225$ & $293 \pm 134$ \\
\hline Min-Max & & 99-414 & $251-465$ & $157-541$ & $238-261$ & $35-600$ & $35-600$ \\
\hline Mean \pm SD & \multirow[t]{2}{*}{$1: 5$} & $388 \pm 1160$ & $383 \pm 1282$ & $526.1 \pm 87.2$ & $386 \pm 98$ & $544 \pm 113$ & $428 \pm 126$ \\
\hline Min-Max & & $165-524$ & $112-519$ & $361-616$ & $203-461$ & $360-651$ & $112-651$ \\
\hline \multicolumn{8}{|c|}{ Phytate: Iron Molar Ratio } \\
\hline Mean \pm SD & \multirow[t]{2}{*}{$1: 2$} & $5.9 \pm 3.2$ & $8.7 \pm 1.6$ & $11.6 \pm 4.8$ & $6.5 \pm 0.3$ & $7.1 \pm 6.9$ & $7.6 \pm 4.0$ \\
\hline Min-Max & & $1.7-12.9$ & $5.9-11.1$ & $4.7-16.1$ & 6.1-6.9 & $0.9-8.6$ & $0.9-18.6$ \\
\hline Mean \pm SD & \multirow[t]{2}{*}{$1: 5$} & $8.6 \pm 3.6$ & $9 \pm 2.9$ & $15.2 \pm 3.1$ & $10.7 \pm 2.6$ & $16.2 \pm 3.8$ & $10.9 \pm 4.4$ \\
\hline Min-Max & & $2.5-13.2$ & $2.9-12.3$ & $9.4-17.9$ & $6.6-13.7$ & $10.3-20.5$ & $2.5-20.5$ \\
\hline & \multicolumn{7}{|c|}{ Phytate: Zinc Molar Ratio } \\
\hline Mean \pm SD & \multirow[t]{2}{*}{$1: 2$} & $6.8 \pm 3.7$ & $12.3 \pm 1.8$ & $13.5 \pm 6.3$ & $7.6 \pm 0.7$ & $9.7 \pm 9.2$ & $9.4 \pm 5.2$ \\
\hline Min-Max & & $1.9-15.4$ & $9.6-14.9$ & $5.3-20.9$ & $6.5-8.4$ & $1.2-23.9$ & $1.2-23.9$ \\
\hline Mean \pm SD & \multirow[t]{2}{*}{$1: 5$} & $11.3 \pm 4.9$ & $13.2 \pm 4.9$ & $21.5 \pm 6.5$ & $12.5 \pm 4.1$ & $22.6 \pm 6.1$ & $14.9 \pm 6.7$ \\
\hline Min-Max & & $3.2-12.9$ & $3.5-18.7$ & $11.7-31.4$ & $6.5-16.6$ & $12.4-28.4$ & $3.2-31.4$ \\
\hline \multicolumn{8}{|c|}{ Ionizable iron } \\
\hline Mean \pm SD & \multirow[t]{2}{*}{$1: 2$} & $0.9 \pm 0.2$ & $1.0 \pm 0.1$ & $0.7 \pm 0.1$ & $0.9 \pm 0.1$ & $0.9 \pm 0.1$ & $0.9 \pm 0.2$ \\
\hline Min-Max & & $0.7-1.2$ & $0.9-1.6$ & $0.7-0.9$ & $0.8-1.0$ & $0.8-1.0$ & $0.7-1.2$ \\
\hline Mean \pm SD & \multirow[t]{2}{*}{$1: 5$} & $1.1 \pm 0.3$ & $1.0 \pm 0.1$ & $0.8 \pm 0.1$ & $0.9 \pm 0.1$ & $0.9 \pm 0.1$ & $1.0 \pm 0.2$ \\
\hline Min-Max & & $0.8-1.6$ & $0.9-1.1$ & $0.7-1.0$ & $0.8-0.9$ & $0.6-1.0$ & $0.7-1.6$ \\
\hline \multicolumn{8}{|c|}{ Extractable Zinc } \\
\hline Mean \pm SD & \multirow[t]{2}{*}{$1: 2$} & $2.8 \pm 0.8$ & $2.1 \pm 0.3$ & $2.6 \pm 0.3$ & $2.3 \pm 0.4$ & $1.9 \pm 0.3$ & $2.3 \pm 0.6$ \\
\hline Min-Max & & $1.8-4.1$ & $1.7-2.5$ & $1.8-2.5$ & $2.1-3.1$ & $1.5-2.1$ & $1.5-4.1$ \\
\hline
\end{tabular}




\begin{tabular}{c|c|c|c|c|c|c|c}
\hline Mean \pm SD & \multirow{2}{*}{$\mathbf{1}: 5$} & $2.9 \pm 0.8$ & $2.1 \pm 0.4$ & $1.8 \pm 0.3$ & $2.3 \pm 0.4$ & $1.7 \pm 0.2$ & $2.3 \pm 0.7$ \\
\cline { 1 - 7 } Min-Max & & $1.7-4.1$ & $1.7-2.8$ & $1.3-2.3$ & $2.0-3.0$ & $1.6-2.0$ & $1.3-4.1$ \\
\hline
\end{tabular}

Table 5. Total and ionizable iron, total and extractable zinc and phytate content, phytate: mineral molar ratios in malted pearl millet $(\mathrm{mg} / 100 \mathrm{gm} \mathrm{DM})$

\begin{tabular}{|c|c|c|c|c|c|c|c|}
\hline \multirow[t]{2}{*}{ Parameter } & \multirow{2}{*}{$\begin{array}{c}\text { Hours, } \\
\text { Grain: Water } \\
\text { Ratio }\end{array}$} & \multicolumn{5}{|c|}{ Variety } & \multirow[t]{2}{*}{ Overall } \\
\hline & & \begin{tabular}{|c|} 
MAHYCO \\
204 \\
\end{tabular} & $\begin{array}{c}\text { ICTP } \\
8203\end{array}$ & H6M33 & $\begin{array}{c}\text { 8203-Mahabeej } / \\
\text { Nirmal }\end{array}$ & Mahalakshmi & \\
\hline \multicolumn{8}{|c|}{ Total Iron } \\
\hline Mean \pm SD & \multirow[t]{2}{*}{$1: 2$} & $4.4 \pm 1.3$ & $3.6 \pm 0.4$ & $3.1 \pm 0.2$ & $3.3 \pm 0.4$ & $3.0 \pm 0.2$ & $3.7 \pm 1.0$ \\
\hline Min-Max & & $2.8-6.3$ & $3.2-4.4$ & $2.8-3.23$ & $2.6-3.7$ & $2.8-3.2$ & $2.6-6.3$ \\
\hline Mean \pm SD & \multirow[t]{2}{*}{$1: 5$} & $4.5 \pm 1.3$ & $3.8 \pm 0.4$ & $3.0 \pm 1.2$ & $3.0 \pm 0.2$ & $2.9 \pm 0.1$ & $3.7 \pm 1.1$ \\
\hline Min-Max & & $2.8-6.4$ & $3.7-4.3$ & $2.8-3.1$ & $2.8-3.3$ & $2.8-3.0$ & $2.6-6.4$ \\
\hline \multicolumn{8}{|c|}{ Total Zinc } \\
\hline Mean \pm SD & \multirow[t]{2}{*}{$1: 2$} & $3.8 \pm 1.2$ & $3.0 \pm 0.6$ & $2.6 \pm 0.3$ & $2.9 \pm 0.4$ & $2.6 \pm 0.3$ & $3.1 \pm 0.9$ \\
\hline Min-Max & & $2.4-5.3$ & $2.5-4.4$ & $2.3-2.9$ & $2.2-3.6$ & $2.1-2.9$ & $2.1-5.3$ \\
\hline Mean \pm SD & \multirow[t]{2}{*}{$1: 5$} & $3.8 \pm 1.3$ & $2.1 \pm 0.5$ & $2.5 \pm 0.5$ & $2.8 \pm 0.6$ & $2.2 \pm 0.4$ & $3.0 \pm 1.0$ \\
\hline Min-Max & & $2.3-5.6$ & $2.2-3.7$ & $1.9-3.1$ & $2.2-3.9$ & $2.0-2.9$ & $1.9-5.6$ \\
\hline \multicolumn{8}{|c|}{ Phytate } \\
\hline Mean \pm SD & \multirow[t]{2}{*}{$1: 2$} & $323 \pm 111$ & $404 \pm 63$ & $353 \pm 106$ & $184 \pm 23$ & $385 \pm 89$ & $331 \pm 111$ \\
\hline Min-Max & & $160-505$ & $283-478$ & $236-510$ & 139-204 & $320-536$ & $139-536$ \\
\hline Mean \pm SD & \multirow[t]{2}{*}{$1: 5$} & $294 \pm 86$ & $313 \pm 68$ & $467 \pm 92$ & 271124 & $474 \pm 125$ & $344 \pm 121$ \\
\hline Min-Max & & $197-506$ & $209-402$ & $313-566$ & $102-471$ & $269-562$ & $102-566$ \\
\hline \multicolumn{8}{|c|}{ Phytate: Iron Molar Ratio } \\
\hline Mean \pm SD & \multirow[t]{2}{*}{$1: 2$} & $7.2 \pm 3.7$ & $9.7 \pm 1.8$ & $9.9 \pm 3.6$ & $4.8 \pm 0.9$ & $10.8 \pm 2.0$ & $8.2 \pm 3.4$ \\
\hline Min-Max & & $2.3-13.7$ & $6.7-11.8$ & $6.0-15.6$ & $3.2-6.0$ & $9.0-14.0$ & $2.3-15.6$ \\
\hline Mean \pm SD & \multirow[t]{2}{*}{$1: 5$} & $6.4 \pm 3.1$ & $7.5 \pm 1.7$ & $12.9 \pm 2.4$ & $7.3 \pm 4.5$ & $13.6 \pm 4.1$ & $8.7 \pm 4.2$ \\
\hline Min-Max & & $2.9-13.2$ & $4.9-9.6$ & $8.5-14.9$ & $2.4-15.6$ & $6.9-17.2$ & $2.4-17.2$ \\
\hline \multicolumn{8}{|c|}{ Phytate: Zinc Molar Ratio } \\
\hline Mean \pm SD & \multirow[t]{2}{*}{$1: 2$} & $9.7 \pm 5.2$ & $13.6 \pm 2.8$ & $13.6 \pm 3.9$ & $6.4 \pm 1.5$ & $14.8 \pm 3.6$ & $11.3 \pm 4.8$ \\
\hline Min-Max & & $3.1-18.5$ & $9.9-17.9$ & $9.6-20.8$ & $3.8-8.4$ & $10.9-19.2$ & $3.1-20.8$ \\
\hline Mean \pm SD & \multirow[t]{2}{*}{$1: 5$} & $8.6 \pm 3.9$ & $10.6 \pm 2.8$ & $18.1 \pm 3.4$ & $9.0 \pm 4.0$ & $18.4 \pm 5.7$ & $11.8 \pm 5.6$ \\
\hline Min-Max & & $3.8-17.8$ & $7.9-15.1$ & $13.5-23.0$ & $4.6-16.2$ & $9.4-24.2$ & $3.8-24.2$ \\
\hline \multicolumn{8}{|c|}{ Ionizable iron } \\
\hline Mean \pm SD & \multirow[t]{2}{*}{$1: 2$} & $1.9 \pm 0.5$ & $1.5 \pm 0.2$ & $1.4 \pm 0.1$ & $1.5 \pm 0.2$ & $1.3 \pm 0.2$ & $1.6 \pm 0.4$ \\
\hline Min-Max & & $1.2-2.8$ & $1.3-1.9$ & $1.2-1.6$ & $1.1-1.7$ & $1.1-1.5$ & $1.1-2.8$ \\
\hline Mean \pm SD & \multirow[t]{2}{*}{$1: 5$} & $2.3 \pm 0.7$ & $2.0 \pm 0.2$ & $1.5 \pm 0.1$ & $1.6 \pm 0.1$ & $1.4 \pm 0.1$ & $1.91 \pm 0.6$ \\
\hline Min-Max & & $1.5-3.2$ & $1.7-2.2$ & $1.4-1.7$ & $1.4-1.8$ & $1.4-1.5$ & $1.4-3.2$ \\
\hline \multicolumn{8}{|c|}{ Extractable Zinc } \\
\hline Mean \pm SD & \multirow[t]{2}{*}{$1: 2$} & $3.0 \pm 1.0$ & $2.3 \pm 0.5$ & $1.9 \pm 0.2$ & $2.3 \pm 0.4$ & $1.9 \pm 0.2$ & $2.4 \pm 0.8$ \\
\hline Min-Max & & $1.9-4.4$ & $1.9-3.4$ & $1.7-2.2$ & $1.6-2.7$ & $1.5-2.1$ & $1.5-4.4$ \\
\hline Mean \pm SD & \multirow[t]{2}{*}{$1: 5$} & $3.1 \pm 1.0$ & $2.4 \pm 0.4$ & $2.1 \pm 0.5$ & $2.4 \pm 0.5$ & $1.8 \pm 0.3$ & $2.5 \pm 0.8$ \\
\hline Min-Max & & $2.00-4.6$ & $1.8-3.0$ & $1.5-2.7$ & $2.00-3.2$ & $1.5-2.3$ & $1.5-4.6$ \\
\hline
\end{tabular}




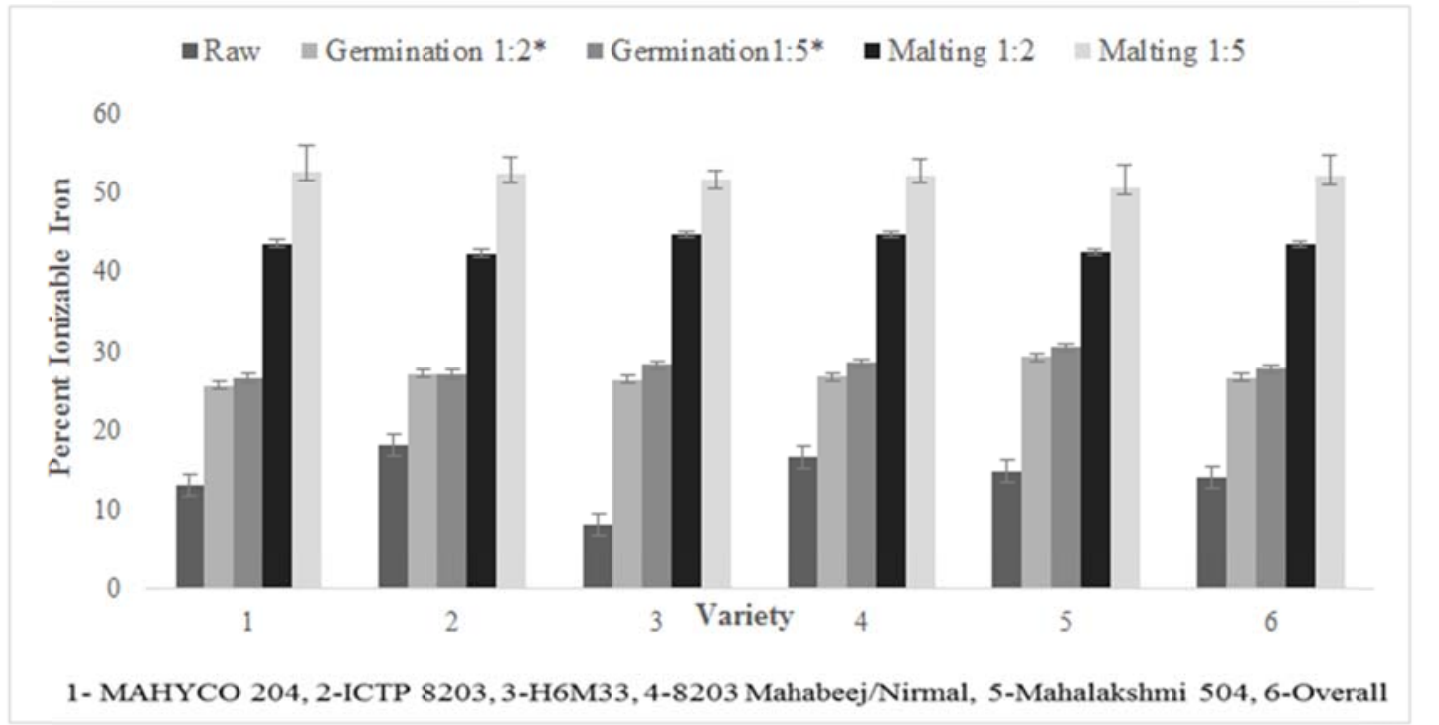

*1:2 and 1:5 represent grain: water ratios used for soaking

Figure 3. Percent ionizable iron in five varieties of raw, germinated and malted pearl millet

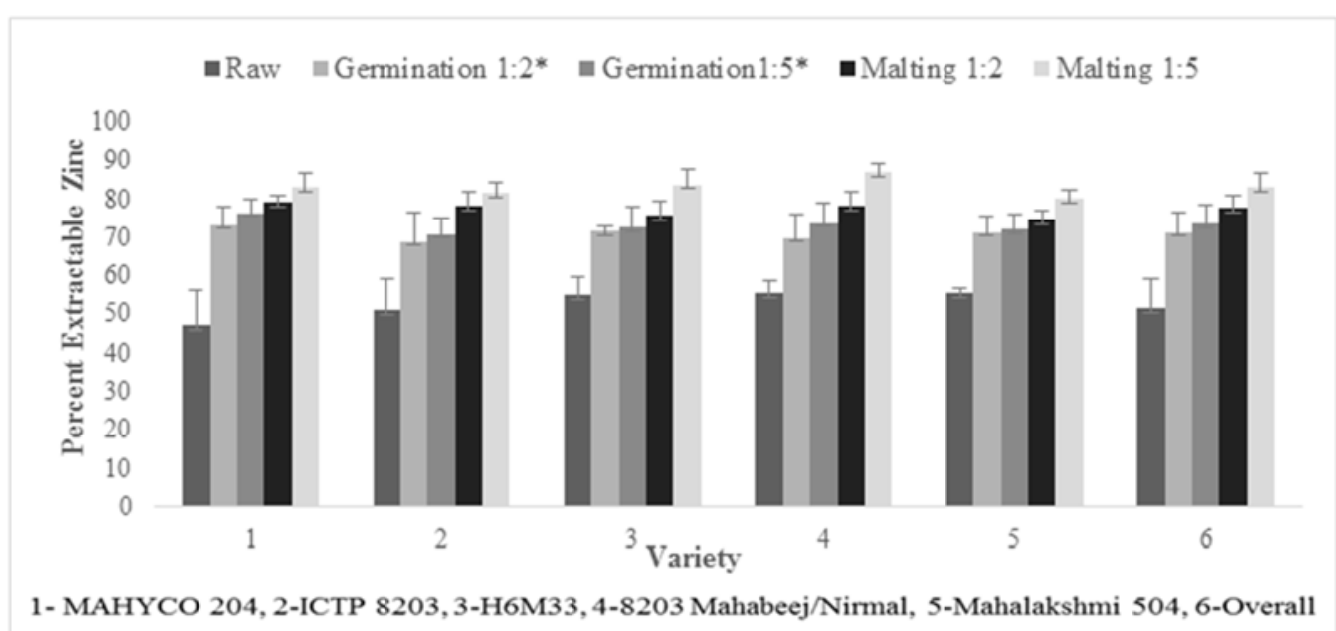

*1:2 and 1:5 represent grain: water ratios used for soaking

Figure 4. Percent extractable zinc in five varieties of raw, germinated and malted pearl millet

\section{Discussion}

The total iron content of pearl millet observed in the present study was compared well with the range of values reported in literature [1, 10-13]. Velu, Rai and Sahrawat [14] also found that ICTP-8203 had the highest iron content in different varieties. However, values observed in the present study were lower than those reported by researchers from other countries such as Sudan $[4,15,16]$, Nigeria $[17,18]$ and the UAE [19].

The total zinc content observed in the present study was similar to values reported by Velu, Rai and Sahrawat [14], who reported zinc content to range from 2.7 to $5.7 \mathrm{mg}$ per 100 grams in 68 varieties of pearl millet, among which ICTP-8203 had a higher zinc content (4.7 mg\%). Similarly, in the present study, the same variety had a slightly higher mean zinc content $(5.2 \mathrm{mg} \%)$ than the other four varieties examined. However, Dave et al., [20] and Sridevi, Yennagi and Basarkar [21] reported very low contents 
(0.022 $\mathrm{mg} \%$ and $0.73 \mathrm{mg} \%$, respectively). Researchers from other countries (Lestienne et al., [2], Eyzaguirre et al., [22], Ahmed et al., [15] and Abdalla et al., [16]) have reported values in the range of 2.51-7.29 mg/100gm whereas Abdelrahman et al., [4] reported slightly lower values (1.26 - 1.79 $\mathrm{mg} / 100 \mathrm{gm})$. Differences between values observed for both iron and zinc in the present study and those reported by other researchers may be due to different geographic locations and/or different varieties.

Soaking has been reported to result in loss of total iron and zinc $[2,15,22]$. In the present study, content of these two minerals decreased after soaking, the losses ranging from approximately 20 to 40 percent, with losses being higher as soaking time increased from 12 to 24 hours and in case of iron also, with use of more amount of water for soaking i.e. grain: water ratio of 1:5. Afify et al., [5] reported losses of iron between $28.1 \%$ and $40.6 \%$ upon soaking sorghum. Loss of these minerals may be attributed to leaching of the ions into the soaking medium due to a concentration gradient [2]. Both iron and zinc are present in the pericarp, aleurone layer and germ of the grain [23]. Lestienne et al., [2] stated that the extent of leaching out of zinc is lower than that of iron and suggested that this may be because iron and zinc are not located in the same part of the seeds and are not linked with the same molecules.

In the present study, germination did not reduce the total iron content to the extent reported by other investigators $[5,15,17$ ]. Eyzaguirre et al., [22] observed $50 \%$ reduction in total iron content of pearl millet after germination for 4 days. Ahmed et al., [15] found that germination for 3 days reduced the iron content of pearl millet by approximately $2-3 \mathrm{mg}$. In the present study, the loss upon germination was $8.8 \%$ to $17.5 \%$. This may be because the period of germination in the present study was short, i.e. 48 hours, compared to other investigators who studied the effects of germination for longer periods up to 96 hours.

Similarly, germination reduced the total zinc content. However, the extent of loss of zinc was greater than that of iron $-31.2 \%$ to $41.7 \%$. The lower content in the germinated grain is largely due to the loss that occurred during soaking, as the content of these two minerals in the germinated samples was not significantly lower than in the soaked samples.

In the present study, malting did not significantly affect iron content compared to the soaked and germinated samples. Abdelrahman et al., [24] reported a minor, non-significant increase in the iron content of malted pearl millet and cereals, whereas other researchers $[25,26]$ have reported decreased total iron content of malted finger millet, wheat and barley. A significant loss of $40 \%$ in the total iron content of pearl millet was reported by Rao and Deosthale [27]. In case of zinc, malted samples had lower content compared to raw samples but did not differ from germinated samples, indicating that the last two steps in the process of malting i.e. dehydration and roasting had no effect on the total iron and zinc content.

Phytate content of the raw grains of the five varieties varied considerably. Cheik et al., [28] also observed a wide range of phytic acid content $(540-1430 \mathrm{mg} / 100 \mathrm{gm})$ in fourteen different cultivars of pearl millet from Burkina Faso. Other researchers, Eltayeb et al., [3], Abdelrahman et al., [4], Sankhala et al., [39], Lestienne et al., [2,40] have reported phytic acid content in the range of values observed in the present study.

Phytate content decreased significantly after each processing. After soaking, the percent reduction was approximately $29 \%$, varying from $23.6 \%$ to $32.4 \%$. Similar trends were observed by other investigators $[2,3,5,31]$. The reduction in phytate content caused by soaking may be due to solubilisation of phytic acid salts in water because it is stored in relatively water soluble form such as sodium and potassium phytate $[5,32,33]$. Also, phytate is present in the aleurone layer and pericarp and hence may leach out into the water used for soaking [34]. Duhan et al.,[35] attributed the loss of phytate in Pigeon pea to leaching out of phytate ions into the soaking water under the influence of a concentration gradient. Further, Bartnik and Szafranska [36] suggested that soaking might activate endogenous phytases depending on the cereal/legume, $\mathrm{pH}$ and temperature. Besides activation of intrinsic phytases, Hotz and Gibson [37] suggested that there may be de novo synthesis of phytase. The extent of phytate reduction depends on the species, $\mathrm{pH}$, length and conditions of soaking $[4,37]$.

Germination has also been found to reduce phytate $[5,38,39]$. In the present study, germination resulted in additional loss of approximately 50\%, compared to soaking. This decrease is attributable to the increased phytase activity during germination. Hydrolysis of phytate due to phytase activity is a step-wise process, with the phosphate being released through the formation of intermediates -inositol pentaphosphate (IP5), inositol tetraphosphate (IP4), inositol tri (IP3), di (IP2) and monophosphates. The final products are inorganic phosphorus, inositol and its intermediate forms of phytate, some of 
which have poor capacity to bind minerals [40,41].

Malting also reduced the phytate content. Malted grains had $60 \%$ lower phytate content compared to the soaked samples, but the difference between malted and germinated samples was only $6.5 \%$. As suggested for germination, the decrease in phytate content can be attributed to stimulation of phytase activity, through either de novo synthesis and/or activation of intrinsic phytases and formation of intermediate forms of phytate $[37,42]$. In the present study, malting involved roasting the germinated grains. Embaby [43] reported that roasting resulted in reduction of phytic acid in peanuts and brown/white sesame seeds; that was attributed to formation of insoluble phytate-protein and /or phytate-protein -mineral complexes. The reduction in phytate with heating could also result from the heat labile nature of phytic acid.

In the present study, we analysed the grains for ionizable iron content at $\mathrm{pH} 7.5$, which is close to the $\mathrm{pH}$ of human duodenum, the site of iron absorption. The values for percent ionizable iron in raw samples obtained in the present study are in the range of values reported in the literature $[4,6,8,10,11,13,17,44]$. Rao and Prabhavathi [8] and Das et al., [11] who used the same method as was used in the present study reported that percent ionizable iron was $7.1 \%$ and $13.75 \%$, respectively. Other researchers $[4,16,17,44]$ who used percent HCL extractable iron, found it to be $18.5-55.2 \%$. Agte et al., [10] and Suma and Urooj [13] analyzed the percent dialyzable iron in raw pearl millet to predict iron availability and found it was $3.2 \%$ and $6.9 \%$ for selected varieties.

Soaking resulted in a slight increase in the ionizable iron content, although the magnitude differed between varieties. Ionizable iron as a percentage of total iron was higher in the soaked than in the raw grains. These findings are in agreement with reports in the literature. Eyzaguirre et al., [22] observed that soaking pearl millet for 24 hours in tap water, increased the percent soluble iron from $30 \%$ to $37 \%$. As observed for ionizable iron, extractable zinc as a percentage of total zinc increased to a small extent after soaking.

Ionizable iron content was $23.3 \%$ more in the germinated grain than in the soaked grain with a grain: water ratio 1:2. However, when the grain: water ratio was 1:5, the ionizable iron content in the germinated grain was only $7.9 \%$ more than the soaked samples. As a percentage of total iron, it constituted about one-fourth. In contrast, the effect on extractability of zinc was less i.e. $17 \%$ when the grain: water ratio was $1: 2$ and $9.5 \%$ when it was 1:5. Malting increased the ionizability of iron substantially, the ionizable iron content being about two times more than in the raw and soaked samples and about $75 \%$ more than in the germinated samples.

Thus, ionizable iron as a percent of total iron was more than $40 \%$ indicating that malting has better potential to improve bioavailability of iron than do soaking and germination. However, malting did not greatly increase the extractability of zinc. The possible reasons for the increase in ionizable iron are the decrease in phytate content as a result of endogenous phytase activity $[5,22]$ and possible reduction in other antinutrients like saponins and phenols $[42,45]$.

Our results on the correlation between the contents of ionizable iron, extractable zinc and phytate support this. We observed strong negative correlation between phytate and ionizable iron, extractable zinc, respectively, indicating that reduction in phytate is an important determinant for improving the ionizability / extractability of these two minerals and thereby their bioavailability. However, the extent of increase in ionizable iron upon malting was more than the reduction in phytate content, suggesting that besides phytate degradation, there may be a positive effect on other inhibitory factors.

There was a significant negative correlation between the ionizable iron content and phytate:iron molar ratio and between the extractable zinc content and phytate:zinc molar ratio. It has been recommended that the phytate: iron molar ratio should be less than one and that of phytate:zinc should be less than $15[46,47]$. In the present study, although germination and malting were effective, they could not lower the ratio below the critical limit. This suggests that there is a need to lower the phytate content further. Greiner [48] reported that if soaking is carried out at 45 to $65^{\circ} \mathrm{C}$ at $\mathrm{pH} 5$ to 6 , as the optimum $\mathrm{pH}$ for endogenous phytase in cereals is 5.15 , phytate dephosphorylation may be favoured. Thus, the conditions of soaking specifically in terms of the $\mathrm{pH}$ and temperature combination need to be optimized.

\section{Conclusion}

Results of this study indicate that availability of iron, zinc can be improved by simple domestic methods 
i.e soaking, germination and malting. For both germination and malting, soaking is a preliminary step which decreases phytate and improve ionizable iron and extractable zinc. However, germination and malting improved these beyond the effect of soaking. Hence, these two processing steps may be recommended specially for foods which are prepared for younger children. Optimum time for soaking appears to be 12 hours with grain:water ratio 1:2 being preferable and also germination for 48 hours appears to be suitable. These simple techniques have great potential for scaling up.

Conflict of Interest. None

Acknowledgements. The authors are grateful to Harvest Plus for financial support and Mr. Anil Udawant, Field Coordinator for procurement of samples.

\section{References}

1. C. Gopalan, B. Sastri, S. Balasubramaniam, et al, Nutritive Value of Indian Foods. National Institute of Nutrition, Indian Council of Medical Research, Hyderabad, 2007.

2. I. Lestienne, C. Icard-Vernière, C. Mouquet, et al, "Effects of soaking whole cereal and legume seeds on iron, zinc and phytate contents," Food Chemistry, vol. 89, pp. 421-425, 2005, doi: 10.1016/j.foodchem.2004.03.040

3. M. Eltayeb, A. Hass, M. Suleiman and E. Babiker, "Effect of processing followed by fermentation on antinutritional factors content of pearl millet (Pennisetum glaucum) cultivars," Pakistan Journal of Nutrition, vol. 6, pp. 463-467, 2007.

4. S. Abdelrahman, H. ELmaki, W. Idris, et al, "Antinutritional factors content and minerals availability of pearl millet (Pennisetum glaucum) as influenced by domestic processing methods and cultivar," Journal of Food Technology, vol. 3, pp. 397-403, 2005.

5. A. Afify, H.S. El-Beltagi, S.M. Abd El-Salam and A.A.Omran, "Bioavailability of iron, zinc, phytate and phytase activity during soaking and germination of white sorghum varieties," PLoS ONE 6:e25512, 2011, doi: 10.1371/journal.pone.0025512.

6. AOAC, Official Method of Analysis, 13th ed. Association of Official Analytical Chemist, Washington, DC, 1980.

7. W. Haug and H-J. Lantzsch, "Sensitive method for the rapid determination of phytate in cereals and cereal products," J Sci Food Agric, vol. 34, pp. 1423-1426, 1983, doi: 10.1002/jsfa.2740341217.

8. B. S. Rao Narasinga and T. Prabhavathi, "An in vitro method for predicting the bioavailability of iron from foods," Am J Clin Nutr, vol. 31, pp. 169-175, 1978.

9. P.T. Chompreeda and M. L. Fields, "Effects of heat and fermentation on the extractability of minerals from soybean meal and corn meal blends," Journal of Food Science, vol. 49, pp. 566-568,1984, doi: 10.1111/j.13652621. 1984. tb12469. x.

10.V.V. Agte, M. K. Gokhale, K.M. Paknikar and S.A. Chiplonkar, "Assessment of pearl millet vs rice based diets for bioavailability of four trace metals," Plant Food Hum Nutr, vol.48, pp.149-158, 1995, doi: 10.1007/BF01088311.

11.P. Das, N. Raghuramulu and K. Rao, "Determination of in vitro availability of iron from common foods,"Journal of Human Ecology, vol. 18, pp. 13-20, 2005.

12.S.K. Gupta, G.Velu, K.N. Rai and K. Sumalini, "Association of grain iron and zinc content with grain yield and other traits in pearl millet (Pennisetum glaucum (L.) R. Br.)," Crop Improvement, vol. 36, pp. 4-7, 2009.

13.P.F. Suma and A. Urooj, "Influence of germination on bioaccessible iron and calcium in pearl millet (Pennisetum typhoideum)," Journal of Food Science and Technology, vol.51, pp. 976-981, 2014, doi: 10.1007/s13197-011-05858

14.G. Velu, K.N. Rai and K.L. Sahrawat, "Variability for grain iron and zinc content in a diverse range of pearl millet populations," Journal of Crop Improvement, vol. 35, pp. 186-191, 2008.

15.A. Ahmed, A. Abdalla and A. El Tinay, "Effect of traditional processing on chemical composition and mineral content of two cultivars of pearl millet (Pennisetum glaucum)," Journal of Applied Sciences Research, vol.5, pp. 2271-2276, 2009.

16.A.A, Abdalla, A.I. Ahmed and A.H El Tinay, "Influence of traditional processing on minerals HCl-extractability of pearl millet (Pennisetum glaucum)," Research Journal of Agriculture and Biological Sciences, vol. 6, pp. 530$534,2010$. 
17.M.H.Badau, I. Nkama and I.A. Jideani, " Phytic acid content and hydrochloric acid extractability of minerals in pearl millet as affected by germination time and cultivar," Food Chemistry, vol. 92, pp. 425-435, 2005, doi: 10.1016/j.foodchem.2004.08.006.

18.F. Sade, "Proximate, antinutritional factors and functional properties of processed pearl millet (Pennisetum glaucum)," Journal of Food Technology, vol. 7, pp. 92-97, 2009.

19.S. Ragaee, E. Abdelaal and M. Noaman, "Antioxidant activity and nutrient composition of selected cereals for food use," Food Chemistry, vol. 98, pp. 32-38, 2006. doi: 10.1016/j.foodchem.2005.04.039

20.S. Dave, B. Yadav and J. Tarafdar, "Phytate phosphorus and mineral changes during soaking, boiling and germination of legumes and pearl millet," Journal of Food Science and Technology, vol. 45, pp. 344-348, 2008.

21.Sridevi, N. Yenagi and P. W. Basarkar, "Antioxidant contents of whole grain cereals of North Karnataka," Karnataka Journal of Agricultural Sciences, vol. 21, pp. 602-3, 2010.

22.R.Z. Eyzaguirre, K. Nienaltowska, L. E. de Jong et al, "Effect of food processing of pearl millet (Pennisetum glaucum) IKMP-5 on the level of phenolics, phytate, iron and zinc", J Sci Food Agric, vol. 86, pp. 1391-1398, 2006. doi: $10.1002 /$ jsfa.2527.

23.J. Taylor, Millet Pearl. Encyclopedia of Grain Science, Elsevier, Oxford, pp. 253-261, 2004.

24.M. AbdelRahaman, B. ElMaki, E. Babiker and A. El Tinay, "Effect of malt pretreatment followed by fermentation on antinutritional factors and HCL extractability of minerals of pearl millet cultivars," Journal of Food Technology, vol. 3, pp. 529-534, 2005.

25.R. Krishnan, U. Dharmaraj and N.G. Malleshi, "Influence of decortication, popping and malting on bioaccessibility of calcium, iron and zinc in finger millet," LWT - Food Science and Technology, vol.48, pp. 169174, 2012, doi: 10.1016/j.lwt.2012.03.003.

26.K. Platel , S.W. Eipeson and K. Srinivasan, "Bioaccessible mineral content of malted finger millet (Eleusinecoracana), wheat (Triticumaestivum), and barley (Hordeumvulgare)," J Agric Food Chem, vol.58, pp. 8100-8103, 2010, doi: 10.1021/jf100846e.

27.D.S. Rao and Y.G. Deosthale, "Mineral composition, ionisable iron and soluble zinc in malted grains of pearl millet and ragi," Food Chemistry, vol. 11, pp. 217-223, 1983, doi: 10.1016/0308-8146(83)90104-8

28.A. Cheik, S. Aly, B. Yaya and T. Alfred, "A comparative study on nutritional and technological quality of fourteen (14) cultivars of pearl millets [Pennisetum glaucum (L) Leeke] in Burkina Faso," Pakistan Journal of Nutrition, vol. 5, pp. 512-521, 2006.

29.A. Sankhala, S. Chopra and A. Sankhala, "Effect of processing on tannin, phytate and in vitro iron in underutilized millets - Bajra Pennisetum typhoideum and KangniSetariaitalica," Indian Journal of Nutrition and Dietetics, vol. 41, pp. 55-62, 2004.

30.I. Lestienne, M. Buisson, V. Lullien-Pellerin, et al, "Losses of nutrients and anti-nutritional factors during abrasive decortication of two pearl millet cultivars (Pennisetum glaucum)," Food Chemistry, vol.100, pp.13161323, 2007. doi: 10.1016/j.foodchem.2005.11.027.

31.V.V. Agte, "Effect of traditional food processing on phytate degradation in wheat and millets" J Agric Food Chem, vol.45, pp. 1659-1661, 1997. doi: 10.1021/jf9605308.

32.R.Chang, S. Schwimmer and H.K. Burr, "Phytate: removal from whole dry beans by enzymatic hydrolysis and diffusion," Journal of Food Science, vol.42, pp.1098-1101, 1977. doi: 10.1111/j.1365-2621. 1977.tb12675. x.

33.P.R. Saha, C. M. Weaver and A.C. Mason, "Mineral bioavailability in rats from intrinsically labeled whole wheat flour of various phytate levels," J Agric Food Chem, vol.42, pp. 2531-2535, 1994, doi: 10.1021/jf00047a029.

34.R.K.Gupta, S.S. Gangoliya and N.K.Singh, "Reduction of phytic acid and enhancement of bioavailable micronutrients in food grains," J Food SciTechnol, pp. 1-9, 2013, doi: 10.1007/s13197-013-0978-y.

35.A. Duhan, N. Khetarpaul and S. Bishnoi, "Content of phytic acid and HCl-extractability of calcium, phosphorus and iron as affected by various domestic processing and cooking methods," Food Chemistry, vol. 78, pp. 9-14, 2002, doi: 10.1016/S0308-8146(01)00144-3.

36.M. Bartnik and I. Szafrańska, "Changes in phytate content and phytase activity during the germination of some cereals," Journal of Cereal Science, vol. 5, pp. 23-28, 1987, doi: 10.1016/S0733-5210(87)80005-X.

37.C. Hotz, and R.S. Gibson, "Traditional food-processing and preparation practices to enhance the bioavailability of micronutrients in plant-based diets," J Nutr, vol. 137, pp. 1097-1100, 2007. 
38.A.Ahmed, A. Abdalla, K. Ibrahim and A. El Tinay, "Effect of traditional processing on phosphorus content and some anti nutritional factors of pearl millet (Pennisetum Glaucum L.)," Research Journal of Agriculture \& Biological Sciences, vol. 6, pp. 176-180, 2010.

39.P.F. Suma and A. Urooj, "Antioxidant activity in two pearl millet (Pennisetum typhoideum) cultivars as influenced by processing," Antioxidants, vol. 3, pp. 55-66, 2014. doi: 10.3390/antiox3010055.

40.O. Ocheme and C. Chinma, "Effects of soaking and germination on some physicochemical properties of millet flour for porridge production," Journal of Food Technology, vol. 6, pp. 185-188, 2008

41.E.A. Mohamed, I. Ahmed and E. Babiker, "Preservation of millet flour by refrigeration: changes in total protein and amino acids composition during storage," WorldAcadSciEngTechnol, vol. 52, pp. 11-14, 2011.

42.C.A. Onyango, S.O. Ochanda, M.A. Mwasaru, et al, " Effects of malting and fermentation on anti-nutrient reduction and protein digestibility of red sorghum, white sorghum and pearl millet," Journal of Food Research, vol. 2, pp. 41, 2013, doi: 10.5539/jfr. v2n1p41.

43.H. Embaby, "Effect of heat treatments on certain antinutrients and in vitro protein digestibility of peanut and sesame seeds," Food SciTechnolRes, vol.17, pp. 31-38. 2011

44.P. Arora, S. Sehgal and A. Kawatra, "Content and HCl-extractability of minerals as affected by acid treatment of pearl millet," Food Chemistry, vol. 80, pp. 141-144, 2003. doi: 10.1016/S0308-8146(02)00379-5.

45.S. Arora, S. Jood and N. Khetarpaul, "Effect of germination and probiotic fermentation on nutrient profile of pearl millet based food blends," British Food Journal, vol. 113, pp. 470-481, 2011. doi: 10.1108/00070701111123952.

46.L. Hallberg, M. Brune and L. Rossander, "Iron absorption in man: ascorbic acid and dose-dependent inhibition by phytate," Am J ClinNutr, vol. 49, pp. 140-144. 1989.

47.J.R. Turnlund, J.C.King, W.R.Keyes, et al, "A stable isotope study of zinc absorption in young men: effects of phytate and alpha-cellulose. Am J ClinNutr, vol. 40, pp. 1071-1077, 1984.

48.R.Greiner and U. Konietzny, "Phytase for food application. FTB journal, vol. 44, pp. 125-140, 2006. 